\title{
Development of additional autoimmune diseases in a population of patients with systemic lupus erythematosus
}

\author{
J E McDonagh, D A Isenberg
}

\begin{abstract}
Objective-In view of the recognised clustering of autoimmune diseases (AID), the chronology of development of other autoimmune diseases in systemic lupus erythematosus (SLE) patients was considered. Methods-A retrospective review of a well documented population of 215 patients with SLE was undertaken. The duration of follow up ranged from 2 to 18 years.

Results-Of these 215 patients, 65 (30\%) had at least one other AID-including 51 (24\%) having one other AID, 12 (6\%) having two and two (1\%) having three other AID in addition to their SLE. Twelve different autoimmune diseases were identified. The majority of patients developed a further AID after SLE had been diagnosed $(62 \%)$ reflecting the relatively early age of onset of SLE. There was no significant difference in the age of onset of rheumatoid arthritis, Sjögren's syndrome and hypothyroidism that had developed before SLE compared with those who developed these diseases after SLE. However, those who developed autoimmune thrombocytopenia (AITP) before SLE were significantly younger than those who developed AITP after SLE (16.7 $v 38.7$ years respectively, p<0.05).

Conclusions-Physicians caring for SLE patients should remain alert to the possible development of a second AID during follow up. Further well matched casecontrol studies are required to define the exact relation between SLE and other AID.

(Ann Rheum Dis 2000;59:230-232)
\end{abstract}

The association of systemic lupus erythematosus (SLE) with other autoimmune diseases (AID) is well established. ${ }^{1-6}$ In view of the variable age of onset of AID (including SLE), a relatively long period of follow up is required to ascertain an accurate prevalence and chronology of the development of additional AID in SLE patients. Such insights may further our understanding of the pathogenesis of these various AID and their inter-relations. The aim of this study was therefore to determine the chronology of development of other AID in SLE patients.

Methods

A retrospective review of laboratory and clinical records was undertaken on 220 consecutive SLE patients attending the
Bloomsbury Rheumatology Unit who have been followed up prospectively from 1978 to 1996. All patients are seen regularly, interviewed, examined and investigated with respect to both SLE manifestations and additional AID. All of the patients with SLE met four or more of the revised classification criteria of the ACR. ${ }^{7}$ Those patients who met the European consensus criteria for Sjogren's syndrome, ${ }^{8}$ the Bohan and Peter criteria for myositis ${ }^{9}$ and four or more of the ACR revised criteria for rheumatoid arthritis $(\mathrm{RA})^{10}$ were noted. In the case of RA, only typical, seropositive, erosive disease was considered. In addition, those patients deemed to have antiphospholipid syndrome, autoimmune thrombocytopenia purpura (AITP), myasthenia gravis, coeliac disease, autoimmune thyroiditis (AITD) and fibrosing alveolitis, were diagnosed on conventional clinical laboratory or histological criteria, or both. Of these patients, records were unavailable on five patients. All of the patients with SLE met four or more of the revised classification criteria of the ACR. ${ }^{7}$ Those patients who met established criteria for additional AID were noted.

Comparison within and between the SLE population and control populations was analysed using $\chi^{2}$ or Fisher's exact tests and two sample $t$ tests where appropriate.

\section{Results}

Of the 215 patients with SLE, 202 were women $(94 \%)$ and 13 men $(6 \%)$. The mean age at original diagnosis was 30.5 years (range 12 to 78 ). The mean age at follow up was 42.2 years (range 16 to 87 ) with a range of follow up from 2 to 18 years. One hundred and forty six were white $(68 \%)$.

THE PREVALENCE OF AUTOIMMUNE DISEASES IN SLE PATIENTS (TABLE 1)

Sixty five patients (30\%) had one or more AID in addition to SLE, $51(24 \%)$ having one AID, $12(6 \%)$ having two AID and two (1\%) having three AID. When those SLE patients with other AID were compared with those SLE patients without an additional AID, there was no difference in sex or ethnic distribution. There was no significant difference in age between the two groups though the former tended to be older (47 $v 30$ years respectively, $\mathrm{p}>0.05)$. SLE patients with other AID had also been significantly younger at time of presentation of their SLE compared with those patients with isolated SLE (32 $v 41.5$ years respectively, $\mathrm{p}<0.01)$. There were relatively fewer SLE
Accepted for publication 26 November 1999 
Table 1 Prevalence of autoimmune diseases in SLE patients

\begin{tabular}{ll}
\hline Autoimmune disease & $\begin{array}{l}\text { Number of SLE } \\
\text { patients (\% of total } \\
\text { population) }\end{array}$ \\
\hline Sjögren's syndrome & $28(13)$ \\
Rheumatoid arthritis & $12(6)$ \\
Autoimmune thrombocytopenia & $10(5)$ \\
Anti-phospholipid syndrome & $9(4)$ \\
Hypothyroidism & $8(4)$ \\
Polymyositis & $6(3)$ \\
Hyperthyroidism & $4(2)$ \\
Myasthenia gravis & $2(1)$ \\
Coeliac disease & $1(0.5)$ \\
Fibrosing alveolitis & $1(0.5)$ \\
Juvenile chronic arthritis & $1(0.5)$ \\
Chronic active hepatitis & $1(0.5)$ \\
\hline
\end{tabular}

patients of white origin than non-white with $\geqslant$ 1 AID though this was not significant $(\mathrm{OR}=$ $0.6, \mathrm{p}>0.05)$.

CHRONOLOGY OF AID DEVELOPMENT IN SLE PATIENTS

SLE was the first disease to occur in the majority of patients who had additional AID $(n=51$, $78 \%)$. However, in a fifth of patients $(n=15$, $23 \%$ ), the additional AID preceded the development of SLE.

Those patients who developed AITP before the onset of SLE were significantly younger at disease onset than those with SLE who subsequently developed AITP (16.7 $v 28.7$ years respectively, $\mathrm{p}<0.05)$. There was no difference in the mean ages of onset of RA (41.6 $v 42.4$ years, $\mathrm{p}>0.05)$, primary Sjögren's syndrome (39.6 $v 31.2$ years, $\mathrm{p}>0.05)$ and hypothyroidism (37.2 v 29.3 years, $\mathrm{p}>0.05)$ that preceded SLE compared with those patients in whom the AID followed SLE (respectively).

\section{Discussion}

This study confirms previous reports ${ }^{1-6}$ that clustering of both systemic and organ specific AID occurs in patients with SLE with almost a third of patients in this study having at least one other AID (30\%).

The majority of patients with an additional AID (62\%) developed the latter after SLE had been diagnosed, which may merely reflect the relatively early age of onset of SLE. However, it may also suggest that SLE itself predisposes to the development of further AID. In a 10 year follow up of RA patients in multicase RA families, a similar prevalence of patients were observed to have an additional AID (37.5\%). ${ }^{11}$ The only AID in the latter population that was increased compared with population controls however, was AITD and no patient developed SLE. ${ }^{12}$ Furthermore, the RA patients tended to be older at follow up than the SLE patients in this study (RA mean age 57.1 years, range 33 to 77 years). ${ }^{11}$ Therefore, SLE may be particularly prone to developing additional AID during the course of the disease. Well matched case-control studies are required to determine whether this observation is merely an effect of age or related to the disease itself.

The finding that in $18 \%$ of patients, the development of SLE was preceded by another AID suggests that it is not simply SLE itself that predisposes to the development of addi- tional AID. Green et al reported a high incidence of AITD in MLR-lpr/lpr mice that in turn develop a lupus-like disease and have a defect in the fas gene that regulates apoptosis $^{12}$ - that is, different manifestations of autoimmunity but the same immunological defect. Such diversity may be explained by other immunogenetic, environmental and hormonal factors.

The majority of patients had SLE without evidence of any additional AID despite follow up ranging up to 18 years. Such disease stability would be consistent with a primary genetic aetiology but the significant minority $(30 \%)$ who changed their autoimmune profile over time supports a role for environmental or random factors, or both, in the pathogenesis of SLE. Certain chemicals have been reported to induce autoantibodies and lupus-like disease in strains of mice not considered genetically susceptible to autoimmune disease. ${ }^{13}$

SLE does not seem to influence the age at which a second AID develops as there was no difference in the age of onset of RA, Sjögren's syndrome or hypothyroidism in those patients in whom SLE developed first compared with those in whom it followed another AID. AITP however was an exception to this rule (see below).

The finding of hyperthyroidism, AITP and myasthenia gravis antedating SLE was in agreement with previous reports. ${ }^{4} 614$

In this study, all six patients with myositis developed the latter after a median interval of 2.5 years (range 1 to 10 ) following diagnosis of SLE. This is in agreement with previous reports ${ }^{15}$ but in contrast with other reports. ${ }^{16}$

Neither of the two patients who had myasthenia gravis that had occurred before onset of SLE, had had a thymoma. Interestingly, Aarli et $a l^{17}$ reported an increased incidence of non-muscle AID among myasthenia gravis patients with follicular hyperplasia of the thymus but not among myasthenia gravis patients with thymoma.

Vaiopoulos et al reviewed the literature and reported that of 26 reported cases of myasthenia gravis associated with SLE, half had preceded and half had followed the development of SLE. ${ }^{6}$ Interestingly, one patient in this study was noted to have developed SLE after a thymectomy in the absence of myasthenia gravis. In a review of the literature, Ciaccio et al reported 13 cases of SLE that followed thymectomy. ${ }^{18}$ The role of thymectomy and the theoretical subsequent effects on suppressor cell function in the development of SLE has been debated ${ }^{19}$ but controversy remains.

There are various significant methodological issues that should be highlighted. The study population had a mean age of 49 years and was representative of a demographic population also at risk of other diseases, for example, autoimmune thyroid disease. The apparent increase in AITD in SLE patients may therefore simply be on the basis of chance alone. Furthermore, those patients with additional AID tended to be older and were younger at time of onset of SLE and therefore 
may merely have had a longer potential period of "immune disregulation" to develop an AID.

Does the association of SLE with some AID represents an autoimmune overlap or manifestations of the same disease? For example, it may be argued that the patients defined in this study as primary Sjögren's syndrome were simply SLE patients with secondary Sjögren's syndrome. However, all patients with primary Sjögren's syndrome satisfied the European consensus criteria for Sjögren's syndrome ${ }^{8}$ including a positive labial biopsy. Such patients can therefore be classified as having an "overlap syndrome"- that is, the coexistence of two separate diseases.

The association of additional AID in SLE patients is likely to be multifactorial with an interplay of ubiquitous environmental and hormonal (or sex related) factors in genetically predisposed people. Physicians caring for SLE patients should remain alert to the possible development of a second AID during follow up and regular serological testing may be advantageous.

1 Schofield RH. Autoimmune thyroid disease in systemic lupus erythematosus and Sjogren's syndrome. Clin Exp Rheumatol 1996;14:321-30.

2 Moutsopoulos HM, Klippel JH, Pavlidis, Wolf RO, Sweet $\mathrm{JB}$, Steinberg AD, et al. Correlative histologic and serologic findings of sicca syndrome in patients with systemic lupus findings of sicca syndrome in patients with systemithe

3 Taneja V, Singh RR, Malaviya AN, Anand C, Mehra NK. Occurrence of autoimmune disease and relationship of Occurrence of autoimmune disease and relationship of autoantibody expression with HLA phenotype in multicase rheumatoi.
4 Karpatkin S. Autoimmune thrombocytopaenic purpura. Semin Haematol 1985;22:260-88.

5 Komatireddy GR, Marshall JB, Aqel R, Spollen LE, Sharp GC. Association of systemic lupus erythematosus and gluten enteropathy. South Med J 1995;88:673-6.

6 Vaiopoulos G, Sfikakis PP, Kapsimali, Boki k, Panayiotidis P, Aessopas A, et al. The association of systemic lupus erythematosus and myasthenia gravis. Postgrad Med J, 1994;70:741-5.

7 Tan EM, Cohen AS, Fries JF, Masi AT, McShane DJ, Rothfield NF, et al. The 1982 revised criteria for the classification of systemic lupus erythematosus. Arthritis Rheum 1982; 25:1271-7.

8 Vitali C, Bombardieri S, Moutsopoulos HM, Balestrieri G, Bencivelli W, Bernstein RM, et al. Preliminary criteria for the classification of Sjögren's syndrome. Arthritis Rheum 1993;36:340-7.

9 Bohan A, Peter JB. Polymyositis and Dermatomyositis. N Engl J Med 1975;292:344-7, 403-7.

10 Arnett FC, Edworthy SM, Bloch DA, McShane DJ, Fries JF, Cooper NS, et al. The American Rheumatism Association 1987 revised criteria for the classification of rheumatoid arthritis. Arthritis Rheum 1988; 31:315-24.

$11 \mathrm{McDonagh}$ JE. Extended pedigree multicase families in rheumatoid arthritis: a ten year follow-up study. [MD theis]. Newcastle upon Tyne: University of Newcastle upon Tyne, 1997.

12 Green LM, LaBue, Lazarus JP, Colburn KK. Characterisation of autoimmune thyroiditis in MLR-lpr/lpr mice. tion of autoimmune

13 Satoh M, Reeves WH. Induction of lupus-associated autoantibodies in BALB/c mice by intra-peritoneal injecautoantibodies in BALB/c mice by intra-perit
tion of pristane. J Exp Med 1994;180:2341-6.

14 Byron MA, Mowat AG. Thyroid disorders in systemic lupus erythematosus. [Letter]. Ann Rhuem Dis 1987;46:174-5.

15 Foote RA, Kimbrough SM, Stevens JC. Lupus myositis. Muscle Nerve 1982;5:65-8.

16 Tsokos GC, Moutsopoulos HM, Steinberg AD. Muscle involvement in systemic lupus erythematosus. JAMA 1981; 246:766-8.

17 Aarli JA, Gilhus NE, Matre R. Myasthenia gravis with thymoma is not associated with an increased incidence of non-muscle autoimmune disorders. Autoimmunity 1992; non-muscle

18 Ciaccio M, Parodi A, Regora A. Myasthenia gravis and lupus erythematosus. Int J Dermatol 1989;28:317-21.

19 Hang M, Theofilopoulos A, Balderas R, Francis S, Dixon F The effect of thymectomy on lupus-prone mice. J Immunol 1984;132:1809-13. 